\title{
Cloning and expression analysis of cinnamoyl-CoA reductase (CCR) genes in sorghum
}

Jieqin Li, Feifei Fan, Lihua Wang, Qiuwen Zhan, Peijin Wu, Junli Du, Xiaocui Yang, Yanlong YL.L Liu

Cinnamoyl-CoA reductase (CCR) is the first enzyme in the monolignol-specific branch of the lignin biosynthetic pathway. In this research, three sorghum CCR genes including SbCCR1, SbCCR2-1 and SbCCR2-2 were cloned and characterized. Analyses of the structure and phylogeny of the three CCR genes showed evolutionary conservation of the functional domains and divergence of function. Transient expression assays in Nicotiana benthamiana leaves demonstrated that the three CCR proteins were localized in the cytoplasm. The expression analysis showed that the three CCR genes were induced by drought. But in $48 \mathrm{~h}$, the expression levels of SbCCR1 and SbCCR2-2 did not differ between CK and the drought treatment; while the expression level of SbCCR2-1 in the drought treatment was higher than in CK. The expression of the SbCCR1 and SbCCR2-1 genes was not induced by sorghum aphid [ Melanaphis sacchari (Zehntner)] attack, but SbCCR2-2 was significantly induced by sorghum aphid attack. It is suggested that SbCCR2-2 is involved in the process of pest defense. Absolute quantitative real-time PCR revealed that the three CCR genes were mainly expressed in lignin deposition organs. The gene copy number of SbCCR1 was significantly higher than those of SbCCR2-1 and SbCCR2-2 in the tested tissues, especially in stem. The results provide new insight into the functions of the three CCR genes in sorghum. 
1 Cloning and expression analysis of cinnamoyl-CoA reductase (CCR) genes in

3 Jieqin $\mathrm{Li}^{\# *}$, Feifei Fan", Lihua Wang, Qiuwen Zhan, Peijin Wu, Junli Du, Xiaocui Yang,

$4 \quad$ Yanlong Liu

5 College of Agriculture, Anhui Science and Technology University, Fengyang, China

$6 \quad$ \#These authors contributed equally to this work.

7

$8 *$ Corresponding author.

9 e-mail: wlhljq@,163.com

10 Tel: $+86-550-6719202$

11

12

13

14

15

16

17

18

19

20 
Abstract: Cinnamoyl-CoA reductase (CCR) is the first enzyme in the monolignol-specific branch of the lignin biosynthetic pathway. In this research, three sorghum CCR genes including $S b C C R 1, S b C C R 2-1$ and $S b C C R 2-2$ were cloned and characterized. Analyses of the structure and phylogeny of the three CCR genes showed evolutionary conservation of the functional domains and divergence of function. Transient expression assays in Nicotiana benthamiana leaves demonstrated that the three CCR proteins were localized in the cytoplasm. The expression analysis showed that the three CCR genes were induced by drought. But in $48 \mathrm{~h}$, the expression levels of $S b C C R 1$ and $S b C C R 2-2$ did not differ between $C K$ and the drought treatment; while the expression level of SbCCR2-1 in the drought treatment was higher than in CK. The expression of the $S b C C R 1$ and SbCCR2-1 genes was not induced by sorghum aphid [Melanaphis sacchari (Zehntner)] attack, but $S b C C R 2-2$ was significantly induced by sorghum aphid attack. It is suggested that SbCCR2-2 is involved in the process of pest defense. Absolute quantitative realtime PCR revealed that the three CCR genes were mainly expressed in lignin deposition organs. The gene copy number of SbCCR1 was significantly higher than those of SbCCR2-1 and SbCCR2-2 in the tested tissues, especially in stem. The results provide new insight into the functions of the three CCR genes in sorghum.

Keywords: sorghum; CCR; gene cloning; qRT-PCR; phylogenetic analysis 
48

\section{Introduction}

Lignin is a complex aromatic polymer present mainly in the secondary cell walls of vascular plants. It plays an important role in specialized conducting and supporting tissues of plants, facilitating water transport, providing mechanical strength, and defending against biotic and abiotic stress (Bhuiyan et al., 2009; Vermerris et al., 2010; Jin et al., 2014). The lignin biosynthetic pathway has attracted researchers' attention because lignin is a limiting factor in a number of agro-industrial processes, such as chemical pulping, forage digestion, and the conversion of lignocellulosic plant biomass to bioethanol (Poovaiah et al., 2014; Tang et al., 2014).

Lignin is derived from the polymerisation of monomeric subunits known as monolignols. The three main monolignols in grasses are $p$-coumaryl, coniferyl and sinapyl alcohols, which give rise to $p$-hydroxyphenyl $(\mathrm{H})$, guaiacyl $(\mathrm{G})$ and syringyl $(\mathrm{S})$ residues, respectively (Boerjan et al., 2003; Vermerris et al., 2010). The biosynthesis of monolignols requires the action of a series of enzymes.

Cinnamoyl-CoA reductase (CCR) is the first enzyme in the monolignol-specific branch of the lignin biosynthetic pathway, where it converts feruloyl-CoA to coniferaldehyde (Figure 1) (Leple et al., 2007). Genes encoding CCR proteins have been studied in many species including Arabidopsis (Lauvergeat and Lacomme et al., 2001; Xue and Luo et al., 2015), rice (Kawasaki and Koita et al., 2006), tobacco (Chabannes and Barakate et al., 2001), soybean (Luderitz and Grisebach, 1981), poplar tree (Leple and Dauwe et al., 2007), maize (Pichon and Courbou et al., 1998; Tamasloukht and Wong et al., 2011), dallisgrass (Giordano and Liu et al., 2014) and wheat (Ma and Tian, 2005). The down regulation of CCR in transgenic Arabidopsis and tobacco leads to significant reduction of lignin content. The CCR gene family is very diverse in plants (Barakat and Yassin et al., 2011). Multiple homologs of CCR genes involved in different functions can be 
72 present in a same plant. For instance, AtCCR 1 is involved in developmental lignification, while 73 AtCCR2 is for stress and elicitor response (Lauvergeat and Lacomme et al., 2001). It has been 74 demonstrated that different CCR genes play different roles in plant's development. Although 75 many studies have been done on CCR genes in different plants, to date, little work on CCR genes 76 in sorghum has been done.

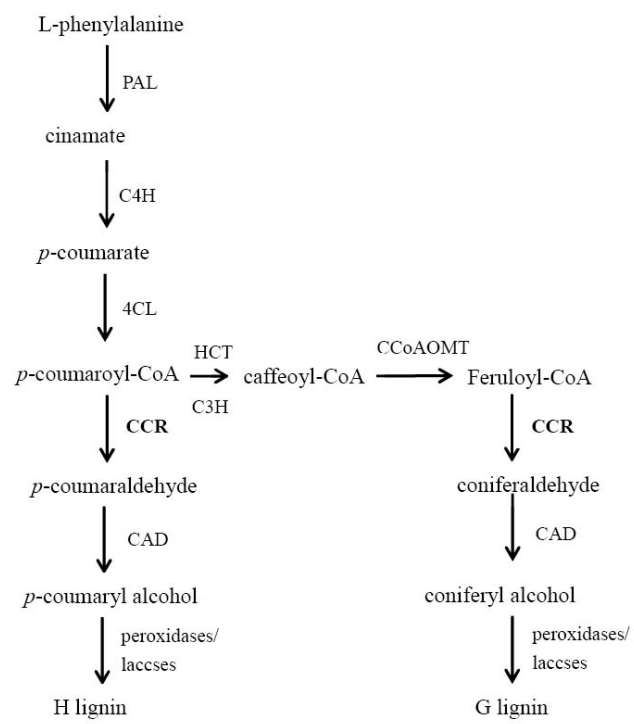

Figure 1 The outline of the lignin biosynthetic pathway. (Abbreviations: $P A L$, phenylalaine ammonia-lyase; $C 4 H$, cinnamate 4-hydroxylase; $4 C L$, 4-coumarate: CoA ligase; $H C T$, $p$-hydroxycinnamoyl-CoA: quinate shikimate $p$-hydroxycinnamoyltransferase; $\mathrm{C} 3 \mathrm{H}$, $p$-coumarate 3-hydroxylase; $C \mathrm{CoAOMT}$, caffeoyl-CoA $O$ methyltransferase; $C C R$, cinnamoyl-CoA reductase; $C A D$, cinnamyl alcohol dehydrogenase)

In this study, three CCR genes were cloned from the cDNA in sorghum seedlings by homology cloning. The structures and functions of their deduced proteins were analyzed by a bioinformatics method. To understand the expression of the three CCR genes in different tissues and treatments, expression analyses were performed. The findings provide a basis for further revealing the roles of the three CCR genes in sorghum.

Materials and methods

Plant materials and gene cloning

Sorghum Tx623B was used for gene cloning and expression analysis. Seeds of Tx623B were pretreated with $75 \%$ alcohol for 2 min and then washed 3 times with distilled water. The 
92

93

94

sterilized seeds were planted in pots. After germination, the seedlings were grown at $28^{\circ} \mathrm{C}$ under $12 \mathrm{~h} \mathrm{light} / 12 \mathrm{~h}$ dark for 2 weeks, taken and ground in liquid $\mathrm{N}_{2}$ for RNA extraction. Total RNA of the seedling samples was extracted using an RNA Prep Pure Plant kit (Tiangen Co., Beijing, China) and was reverse transcribed using a SuperScript II kit (TaKaRa Biomedical, Shiga, Japan).

Cloning primers for the three CCR genes (i.e., Sb07g021680 named SbCCR1, Sb02g014910 named SbCCR2-1, Sb04g005510 named SbCCR2-2) were designed according to the CCR gene sequences in the National Center for Biotechnology Information (NCBI) database. The amplification primers are listed in Table 1. Polymerase chain reaction (PCR) was performed using KOD FX polymerase (Toyobo, Osaka, Japan). The amplified fragments were separated on 1\% agarose gels and purified by DNA Gel Extraction Kit (AxyPrep DNA Gel Extraction kit, Axygen, USA). Then, the purified fragments were linked to pMD18-T vector (Takara, China) and transformed into E. coli DH5a. The recombinant plasmids were verified by PCR; the positive clones were sent to Genscript Company (Nanjing, China) for sequencing.

Table 1 The primers used in the research

Drought and pest defense experiments

For the drought defense experiment, Tx623B seeds were sterilized as described above and germinated on wet paper. After germination, the seedlings were grown at $28^{\circ} \mathrm{C}$ under $12 \mathrm{~h}$ light $/ 12 \mathrm{~h}$ dark for 2 weeks. Then half of the seedlings were treated with $20 \%$ polyethylene glycol (PEG) 6000 as drought treatment and sampled at $24 \mathrm{~h}$ and $48 \mathrm{~h}$. The other half of the seedlings were untreated with PEG 6000 but sampled at the same times as control.

For the pest defense experiment, Tx623B seeds were sterilized, germinated and grown as in the drought defense experiment. Then, for half of the seedlings, 4 pest-attacked sorghum leaves with 2-4 sorghum aphids [Melanaphis sacchari (Zehntner)] on each were put close to each seedling so that the aphids would migrate to the seedlings; subsequently, the seedlings were placed in a cage. After the sorghum seedlings were attacked by the aphids, their pest-attacked leaves were sampled at $24 \mathrm{~h}$ and $48 \mathrm{~h}$ for RNA extraction. The other half of the seedlings was 
120

121

122

used as control and leaves were sampled at the same times for RNA extraction.

Sequence analysis and phylogenetic analysis

Nucleotide sequences were translated into protein sequences which were then aligned using BioEdit. Phylogenetic analyses were performed using the neighbor joining (NJ) method with Mega 5.1 software. Branch support was assessed with 1,000 bootstrap replicates.

Subcellular localization

The primer pairs for the three CCR genes were used to amplify the cDNA fragments encoding the full-length CCR proteins. The PCR fragments for each CCR gene were inserted into the vector $1305 \mathrm{GFP}$ at the N-terminus of the green fluorescent protein (GFP) under the control of cauliflower mosaic virus 35S promoter. An Agrobacterium tumefaciens strain carrying the $35 S:: C C R 1-G F P, 35 S:: C C R 2-1-G F P, 35 S:: C C R 2-2-G F P$ or $35 S:: G F P$ plasmid was infiltrated into Nicotiana benthaminana leaves and analyzed with confocal microscopy $48 \mathrm{~h}$ after agroinfiltration as described previously (Goodin and Dietzgen et al., 2002). Fluorescence of GFP was observed with a Leica LSM710 confocal laser scanning microscope.

Quantitative real-time PCR

Quantitative real-time PCR (qRT-PCR) was performed using a SYBR Green supermix (BioRad, USA) on an ABI prism 7900 real-time PCR system. The primers for the three CCR genes are listed in Table 1. The sorghum eIF4al gene (Sb04g003390) was used as the endogenous control in the experiment. All reactions were run in three replicates. The $2^{-\Delta \Delta C T}$ method was used to analyze relative changes in gene expression (Livak and Schmittgen, 2001).

\section{Absolute qRT-PCR}

The three genes' coding fragments were aligned with pMD18-T vector. Fresh 10-fold serial dilutions were created from the three constructs. The concentration of the plasmid preps was 
147 148

149

150

151

152

153

154

155

156

157

158

159

160

161

162

163

164

165

166

167

168

169

170

171

172

measured using a Biodrop Touch spectrophotometer. The corresponding gene copy number in plasmid was calculated with the following equation:

$$
\text { gene copy number }=\frac{6.02 \times 10^{23}(\mathrm{copy} / \mathrm{mol}) \times \mathrm{DNA} \text { amount }(g)}{\text { DNA length }(\mathrm{bp}) \times 660\left(\frac{\mathrm{g}}{\mathrm{mol}} / \mathrm{bp}\right)}
$$

where DNA length stands for the combined length of plasmid and insert, in base pairs, and DNA amount equals the corresponding plasmid concentration times the volume (Hanzel et al., 2016). The linear regression equation and $R^{2}$ were obtained by comparing the log values of the DNA concentration and the $\mathrm{Ct}$ values. Then, gene copy number was calculated with the corresponding linear regression equation.

\section{Results}

1. Cloning and structure analysis of $S b C C R 1, S b C C R 2-1$ and $S b C C R 2-2$

Based on data obtained from the sorghum genome sequence and research results (Giordano and Liu et al., 2014), 3 CCR genes were identified by PCR and sequencing. Sequence annotation and protein chemical characterization of the three CCR genes are shown in Table 2. The sequencing results showed that the cDNA sequences of the three CCR genes were completely consistent with those in NCBI database. Molecular analysis of the deduced proteins showed that SbCCR1, SbCCR2-1 and SbCCR2-2 contained 374, 346 and 343 amino acids, respectively. Multiple alignments showed that the highest similarity between SbCCR1 and ZmCCR1 was 89.3\% (Figure 2). The similarity between SbCCR2-1 and SbCCR2-2 (59.8\%) was higher than that between SbCCR1 and SbCCR2-1 (58.3\%) and that between SbCCR1 and SbCCR2-2 (49.3\%).

Commonly, a CCR protein contains an NADP binding domain and a catalytic activity domain (NWYCY) (Pan et al., 2014). The multiple alignments showed that SbCCR1, SbCCR2-1 and SbCCR2-2 contained an NADP binding domain, an NWYCY domain and a substrate domain (Figure 2). These results demonstrated that the three CCR genes contained the functional domains of CCR, suggesting that the three CCR proteins should be of CCR activities.

Table 2 Molecular characteristics of CCR genes in Sorghum bicolor 
173

174

175

176

177

178

179

180

181

182

183

184

185

186

187

188

189

190

191

192

193

194

195

196

197

198

199

200

201

202

Figure 2 Multiple alignment of the protein sequences of sorghum CCR proteins and other plant CCR proteins. (The alignment includes sequences from Arabidopsis (AtCCR1 and AtCCR2) and maize (ZmCCR1). The amino acids believed to be part of the active sites are shown below the alignment with the following codes:

$\bullet=$ catalytic activity domain; $\circ=$ NADP binding domain; $\boldsymbol{\square}=$ substrate binding domain.)

2. Phylogenetic analysis of the CCR proteins

Phylogenetic analysis of the CCR proteins encoded in sorghum and other plant genomes showed that there were two major clades (Figure 3). One clade was mainly comprised of CCR1 proteins; the other was mainly comprised of CCR2 proteins. The two clades were supported with moderate bootstrap values. SbCCR1 was included in the first clade; while SbCCR2-1 and SbCCR2-2 were included in the second clade. The clade containing mainly CCR1 proteins was comprised of two major sub-clades: one consisted of dicotyledons (e.g., Glycine max and Arabidopsis thaliana), the other monocotyledons (e.g., Sorghum bicolor, Hordeum vulgare, Lolium perenne, Zea mays and Oryza sativa) and both were supported with high bootstrap values.

Figure 3 Phylogenetic analyses of sorghum CCR proteins and other plant CCR homologues.

3. Subcellular localization of CCR proteins

CCR genes perform their functions in cytoplasm. Therefore, the three CCR proteins were further analyzed with the protein subcellular localization prediction program WoLF PSORT (http://wolfpsort.org/). All of these proteins were predicted to be in cytoplasm. To verify the predicted results, a transient expression assay for the three CCR proteins was performed in $N$. benthamiana leaves. GFP alone was expressed as control, SbCCR1, SbCCR2-1 and SbCCR2-2 in full-length were fused to the N-terminus of GFP. The results showed that free GFP dispersed throughout the cytoplasm in the $N$. benthamiana epidermal cells; and the green fluorescent signal of GFP was not co-located with the auto-fluorescence of chlorophylls in chloroplasts. The localization patterns of SbCCR1-GFP, SbCCR2-1-GFP and SbCCR2-2-GFP were similar to that of free GFP (Figure 4), indicating that the three CCR proteins were targeted to the cytoplasm.

Figure 4 The subcellular localization of sorghum CCR genes in $N$. benthamiana leaves (transient expression 
203

204

205

206

207

208

209

210

211

212

213

214

215

216

217

218

219

220

221

222

223

224

225

226

227

228

229

230

231

of SbCCR1-GFP, SbCCR2-1-GFP and SbCCR2-2-GFP fusion proteins and GFP in N. benthamiana leaves; GFP, fluorescence of SbCCR1-GFP, SbCCR2-1-GFP, SbCCR2-2-GFP and GFP; Auto, Ch1 autofluorescence; Merged, merged images of GFP and Auto ones in bright).

4. Expression analysis of CCR genes in drought and pest treatments

To understand the functions of the three CCR genes in sorghum, expression analysis was performed in drought treatment (Figure 5). The results showed that the expression levels of $S b C C R 1, S b C C R 2-1$ and $S b C C R 2-2$ were significantly higher in the drought treatment than in $\mathrm{CK}$ at $24 \mathrm{~h}$, indicating that all of the three CCR genes were induced by drought. But at $48 \mathrm{~h}$, there were no differences in the expression levels of SbCCRI and SbCCR2-2 between CK and the drought treatment; the expression level of $S b C C R 2-1$ was higher in the drought treatment than in CK. The results suggested that all of these genes were involved in the process of drought defense. $S b C C R 1$ and $S b C C R 2-2$ mainly responded in the first phase of drought defense, while the $S b C C R 2-1$ gene responded during the whole drought defense period.

Figure 5 Expression of sorghum CCR genes in CK and the drought treatment at different times (A, SbCCR1; B, SbCCR2-1; C, SbCCR2-2; variance analysis was used for statistical test; different lowercase letters represent significant difference at 0.05 probability level).

To identify the functions of the three CCR genes in pest defense, expression analysis was performed. The expression levels of the SbCCR1 and SbCCR2-1 genes were not different between $\mathrm{CK}$ and the pest treatment at $24 \mathrm{~h}$ or $48 \mathrm{~h}$ (Figure 6). But the expression levels of the SbCCR2-2 gene at $24 \mathrm{~h}$ and $48 \mathrm{~h}$ in the pest treatment were significantly higher than in CK, indicating that $S b C C R 2-2$ was involved in pest defense.

Figure 6 Expression of sorghum CCR genes in CK and the pest treatment at different times (pest used was sorghum aphids (Melanaphis sacchari (Zehntner)); A, SbCCR1; B, SbCCR2-1; C, SbCCR2-2; variance analysis was used for statistical test; different lowercase letters represent significant difference at 0.05 probability level).

5. Spatial expression patterns of CCR genes 
232

233

234

235

236

237

238

239

240

241

242

243

244

245

246

247

248

249

250

251

252

253

254

255

256

257

258

The linear regression equations and $R^{2}$ values of the three CCR genes were obtained by comparing the log values of DNA concentration and the $\mathrm{Ct}$ values. The slopes of the standard quantification curves for $S b C C R 1, S b C C R 2-1$ and $S b C C R 2-2$ were -3.25, -3.56 and -3.39, respectively. The $R^{2}$ values of the three equations were 0.99 . The gene copy numbers of $S b C C R 1$, $S b C C R 2-1$ and $S b C C R 2-2$ in various plant organs, including leaf, spikelet, stem and root, were quantified using their corresponding linear regression equations. The results demonstrated that the three CCR genes were expressed in allthe tested organs (Figure 7). The expression patterns of the three CCR genes were consistent with the deposition patterns of lignin in these organs. The highest expression levels of the three CCR genes were found in stem. The expression levels of $S b C C R 1$ and $S b C C R 2-2$ in stem and root were obviously higher than in spikelet and leaf, but those of $S b C C R 2-1$ in stem and spikelet were higher than in leaf and root. The gene copy number of $S b C C R 1$ was significantly higher than those of SbCCR2-1 and SbCCR2-2 in root, leaf, stem and spikelet, especially in stem. This indicated that the expression level of SbCCRl was obviously higher than those of SbCCR2-1 and SbCCR2-2 in the tested sorghum tissues.

Figure 7 The copy numbers of CCR genes in different sorghum tissues (variance analysis was used for statistical test; different lowercase letters represent significant difference at 0.05 probability level.)

\section{Discussion}

The biosynthesis of lignin begins with the common phenylpropanoid pathway starting with the deamination of phenylalanine and leading to cinnamoyl-CoA esters which are then channeled into the lignin branch pathway via CCR (Pichon and Courbou et al., 1998). CCR genes were first cloned and characterized in Eucalyptus, providing insight into the regulation of the lignin biosynthetic pathway in developmental and defense processes (Lacombe and Hawkins et al., 1997; Lacombe and Van Doorsselaere et al., 2000). In this research, the molecular and expression characteristics of three CCR genes in sorghum were reported. The results showed that the three CCR genes exhibited different biochemical properties and differential expression patterns in response to drought and pest treatments. 
259

260

261

262

263

264

265

266

267

268

269

270

In higher plants, there are a number of CCR and CCR-like genes (Barakat and Yassin et al., 2011). But only one to two CCR genes (bona fide CCR genes) are involved in lignin biosynthesis during plant development. For example, AtCCRl is the only bona fide CCR gene in Arabidopsis (Mir and Sierra et al., 2008). In this study, the SbCCRl gene showed high sequence similarity with the ZmCCRl gene which is the bona fide CCR gene in maize (Tamasloukht and Wong et al., 2011). Phylogenetic analysis revealed that SbCCR1 was closer to other CCR1 proteins involved in lignin biosynthesis in plant developmental processes. And the expression analysis also showed that the $S b C C R l$ gene was mainly expressed in stem where lignin was deposited at high content. Additionally, the expression level of SbCCRl was significantly higher than those of $S b C C R 2-1$ and $S b C C R 2-2$ in various tissues. This indicated that the $S b C C R 1$ gene should be mainly involved in the biosynthesis of lignin. All these results indicated that $S b C C R 1$ should be a bona fide CCR gene in sorghum.

CCR2 gene is mainly involved in defense-related processes in plant (Fan and Linker et al., 2006). In this research, phylogenetic analysis revealed that $S b C C R 2-1$ and $S b C C R 2-2$ were in a same clade, indicating that the two genes should belong to CCR2 gene. Spatial expression analysis showed that the three CCR genes were mainly expressed in tissues with lignin deposited, but expression analysis of the CCR genes in drought and pest treatments showed that $S b C C R 2-1$ was mainly involved in the process of drought defense, while $S b C C R 2-2$ was involved in pest defense. All these results suggested that $S b C C R 2-1$ and $S b C C R 2-2$ mainly played a role in defense-related processes in sorghum.

In plants, the CCR1 and CCR2 genes show different expression patterns in response to biotic and abiotic stresses (Fan and Linker et al., 2006; Tamasloukht and Wong et al., 2011). For instance, AtCCR 1 and $A t C C R 2$ show different expression patterns in response to pathogen infection. AtCCR 1 transcript level is not significantly changed, but AtCCR2 highly accumulates in infected leaves with Xanthomonas campestris pv. campestris (Lauvergeat and Lacomme et al., 2001). In this research, it was also observed that the transcript levels of SbCCR 1 and $S b C C R 2-1$ were not significantly affected by pest. Whereas, the transcript level of $S b C C R 2-2$ was strongly 
286

287

288

289

290

291

292

293

294

295

296

297

298

299

300

301

302

303

304

305

306

307

308

309

310

311

2

affected by pest attack. It was showed that $S b C C R 2-1$ and $S b C C R 2-2$ played different roles in response to pest attack, suggesting a role of $S b C C R 2-2$ in pest resistance.

\section{CONCLUSIONS}

In this research, three CCR genes were cloned and characterized in sorghum. Phylogenetic analysis revealed that the $S b C C R 1$ gene belongs to one clade, while $S b C C R 2-1$ and $S b C C R 2-2$ belong to another clade. Subcellular localization indicated that the three CCR genes were localized in the cytoplasm. qRT-PCR demonstrated that the three CCR genes were induced by drought. But the expression pattern of $S b C C R 2-1$ was different from those of $S b C C R 1$ and $S b C C R 2-2$. The expressions of the $S b C C R 1$ and $S b C C R 2-1$ genes were not induced by pest attack, but that of $S b C C R 2-2$ was significantly induced by pest. It was suggested that $S b C C R 2-2$ was involved in the process of pest defense. Absolute qRT-PCR analysis showed that the three CCR genes have similar spatial expression patterns. The gene copy number of $S b C C R l$ was significantly higher than those of SbCCR2-1 and SbCCR2-2 in the tested tissues, especially in stem. The results from this study provide important information for understanding the roles of the three CCR genes in sorghum.

3

Barakat A, Yassin NB, Park JS, Choi A, Herr J, Carlson JE. 2011. Comparative and phylogenomic analyses of cinnamoyl-CoA reductase and cinnamoyl-CoA-reductase-like gene family in land plants. Plant Sci 181: 249-257.

Bhuiyan NH, Selvaraj G, Wei Y, King J. 2009. Role of lignification in plant defense. Plant Signal Behav 4: $158-159$.

Boerjan W, Ralph J, Baucher M. 2003. Lignin biosynthesis. Annu Rev Plant Biol 54: 519-546.

Chabannes M, Barakate A, Lapierre C, Marita JM, Ralph J, Pean M, Danoun S, Halpin C, GrimaPettenati J, Boudet AM. 2001. Strong decrease in lignin content without significant alteration of plant 
312 development is induced by simultaneous down-regulation of cinnamoyl CoA reductase (CCR) and 313 cinnamyl alcohol dehydrogenase (CAD) in tobacco plants. Plant J 28: 257-270.

314 Fan L, Linker R, Gepstein S, Tanimoto E, Yamamoto R, Neumann PM. 2006. Progressive inhibition by 315 water deficit of cell wall extensibility and growth along the elongation zone of maize roots is related to 316 increased lignin metabolism and progressive stelar accumulation of wall phenolics. Plant Physiol 140: 317 603-612.

318 Giordano A, Liu Z, Panter SN, Dimech AM, Shang Y, Wijesinghe H, Fulgueras K, Ran Y, Mouradov A, 319 Rochfort S, Patron NJ, Spangenberg GC. 2014. Reduced lignin content and altered lignin composition in 320 the warm season forage grass Paspalum dilatatum by down-regulation of a Cinnamoyl CoA reductase gene. Transgenic Res 23: 503-517.

322 Goodin MM, Dietzgen RG, Schichnes D, Ruzin S, Jackson AO. 2002. pGD vectors: versatile tools for the expression of green and red fluorescent protein fusions in agroinfiltrated plant leaves. Plant $J$ 31: 375-383. Gotia HT, Munro JB, Knowles DP, Daubenberger CA, Bishop RP, Silva JC. 2016. Absolute Quantification of the Host-To-Parasite DNA Ratio in Theileria parva-Infected Lymphocyte Cell Lines. PLoS One 11: e150401.

Jin Y, Zhang C, Liu W, Qi H, Chen H, Cao S. 2014. The cinnamyl alcohol dehydrogenase gene family in melon (Cucumis melo L.): bioinformatic analysis and expression patterns. PLoS One 9: e101730. Kawasaki T, Koita H, Nakatsubo T, Hasegawa K, Wakabayashi K, Takahashi H, Umemura K, Umezawa T, Shimamoto K. 2006. Cinnamoyl-CoA reductase, a key enzyme in lignin biosynthesis, is an effector of small GTPase Rac in defense signaling in rice. Proc Natl Acad Sci US A 103: 230-235. Grima-Pettenati J. 1997. Cinnamoyl CoA reductase, the first committed enzyme of the lignin branch biosynthetic pathway: cloning, expression and phylogenetic relationships. Plant J 11: 429-441. and in response to infection with pathogenic bacteria. Phytochemistry 57: 1187-1195. 
339

340

341

342

343

344

345

346

347

348

349

350

351

352

353

354

355

356

357

358

359

360

361

362

363

364

365

Lefebvre A, Joseleau JP, Grima-Pettenati J, De Rycke R, Andersson-Gunneras S, Erban A, Fehrle I, PetitConil M, Kopka J, Polle A, Messens E, Sundberg B, Mansfield SD, Ralph J, Pilate G, Boerjan W. 2007. Downregulation of cinnamoyl-coenzyme A reductase in poplar: multiple-level phenotyping reveals effects on cell wall polymer metabolism and structure. Plant Cell 19: 3669-3691.

Livak KJ, Schmittgen TD. 2001. Analysis of relative gene expression data using real-time quantitative PCR and the 2(-Delta Delta C(T)) Method. Methods 25: 402-408.

Luderitz T, Grisebach H. 1981. Enzymic synthesis of lignin precursors. Comparison of cinnamoyl-CoA reductase and cinnamyl alcohol:NADP+ dehydrogenase from spruce (Picea abies L.) and soybean (Glycine max L.). Eur J Biochem 119: 115-124.

Ma QH, Tian B. 2005. Biochemical characterization of a cinnamoyl-CoA reductase from wheat. Biol Chem 386: 553-560.

Mir DM, Sierra JB, Ruel K, Pollet B, Do CT, Thevenin J, Buffard D, Jouanin L, Lapierre C. 2008. Redirection of the phenylpropanoid pathway to feruloyl malate in Arabidopsis mutants deficient for cinnamoyl-CoA reductase 1. Planta 227: 943-956.

Pan H, Zhou R, Louie GV, Muhlemann JK, Bomati EK, Bowman ME, Dudareva N, Dixon RA, Noel JP, Wang X. 2014. Structural studies of cinnamoyl-CoA reductase and cinnamyl-alcohol dehydrogenase, key enzymes of monolignol biosynthesis. Plant Cell 26: 3709-3727.

Pichon M, Courbou I, Beckert M, Boudet AM, Grima-Pettenati J. 1998. Cloning and characterization of two maize cDNAs encoding cinnamoyl-CoA reductase (CCR) and differential expression of the corresponding genes. Plant Mol Biol 38: 671-676.

Poovaiah CR, Nageswara-Rao M, Soneji JR, Baxter HL, Stewart CJ. 2014. Altered lignin biosynthesis using biotechnology to improve lignocellulosic biofuel feedstocks. Plant Biotechnol J 12: 1163-1173.

Tamasloukht B, Wong QLM, Martinez Y, Tozo K, Barbier O, Jourda C, Jauneau A, Borderies G, Balzergue S, Renou JP, Huguet S, Martinant JP, Tatout C, Lapierre C, Barriere Y, Goffner D, Pichon M. 2011. Characterization of a cinnamoyl-CoA reductase 1 (CCR1) mutant in maize: effects on lignification, fibre development, and global gene expression. J Exp Bot 62: 3837-3848.

Tang HM, Liu S, Hill-Skinner S, Wu W, Reed D, Yeh CT, Nettleton D, Schnable PS. 2014. The maize 
366 brown midrib2 (bm2) gene encodes a methylenetetrahydrofolate reductase that contributes to lignin 367 accumulation. Plant J 77: 380-392.

368 Vermerris W, Sherman DM, McIntyre LM. 2010. Phenotypic plasticity in cell walls of maize brown 369 midrib mutants is limited by lignin composition. $J$ Exp Bot 61: 2479-2490.

370 Xue J, Luo D, Xu D, Zeng M, Cui X, Li L, Huang H. 2015. CCR1, an enzyme required for lignin 371 biosynthesis in Arabidopsis, mediates cell proliferation exit for leaf development. Plant J 83: 375-387. 


\section{Figure 1 (on next page)}

Figure 1 
L-pHenerglanine

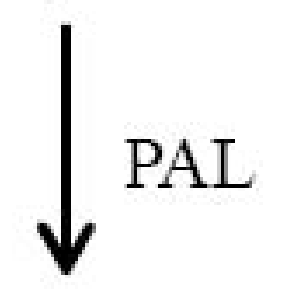

cinamate

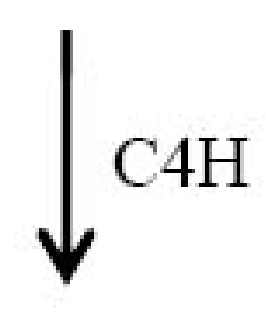

p-coumarate

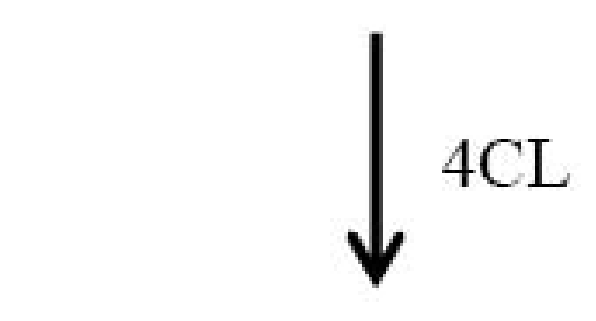

\section{HCT CCOAOMT}

$p$-coumaroyl-CoA $\longrightarrow$ caffeoyl-CoA $\longrightarrow$ Feruloyl-CoA

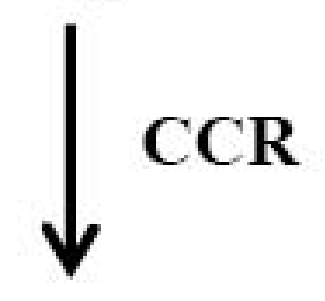

p-coumaraldehyde

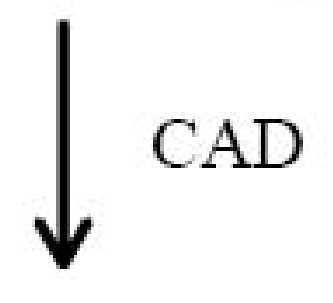

p-coumaryl alcohol

peroxidases/

laccses
$\mathrm{C} 3 \mathrm{H}$

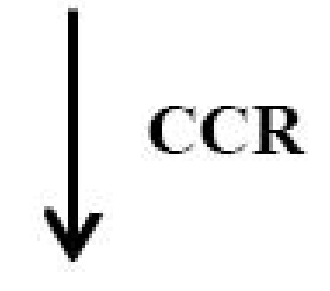

coniferaldehyde

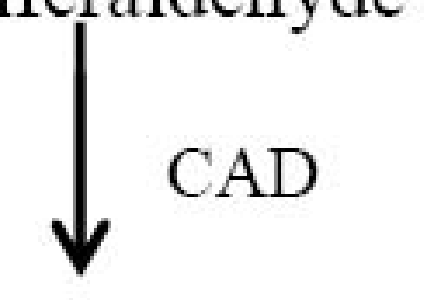

coniferyl alcohol

peroxidases/

laccses 


\section{Figure 2 (on next page)}

Figure 2 
SbCCR1 SbCCR2-1 SbCCR2-2 $\mathrm{ZmCCR} 1$ AtCCR1

AtCCR2

SbCCR1 SbCCR 2-1 SbCCR2-2 ZmCCR1 AtCCR1 AtCCR2

SbCCR1 SbCCR2-1 SbCCR2-2 ZmCCR1 AtCCR1 AtCCR2
MTVVDAVSTDAAGAAPAAAAAPVVVAQPGNGQTVCVTGA AGIASWIVKMLLEKGYTVKGTVRNP-DDPKNAHLK MPTAETTTPVPPALS-----------GQGRIVCVTGAGGFIASWIVKRLLEKGYTVRGTVRNP-VDPKNDHLR - - - - - - - - - - - MTVVDAVVSSTDAGAPAAAAT---AVPAGNGQTVCVTGA AGYIASWIVKILLEKGYTVKGTVRNPADDPKNAHLK -MPVDVASP-----------------AGKIVCVTGAGGYIASW IVKILLERGYTVKGTVRNP-DDPKNIHLR -MLVD-----------------GKIVCVTGAGGYIASW IVKILLERGYTVRGTVRNP-TDPKNNHLR 00000

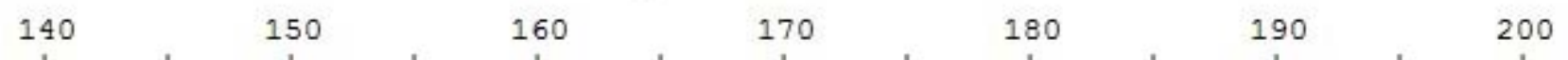

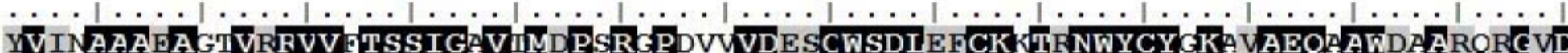
YVMIIAAADT-GVKRVVFTSSIGIIVMN PYR PNKAVDDTCWSDLEYCK TONWYCYAKIVAEQG AWEVARKRGLD NVVEAAADA-GVRRIVLSSTIGIMYMDPRRDPDAALGDSSWSDLEYCK STKNWYCYAKT IAEQ AWEA ARARGLD YVINAAA EA GTVRRVVFTSSIGZVIMDPKRGPDVVVDESCWSDLE FCEKTF NWYCYGKAVAEQA AWEIIARR CVD FVIN AAAEA KVKRVVITSSIGA VWMDPNR PEAVVDESCWSDLD FK TKNWYCY GK VVAEQA AWE IAKEKCVD FVIDAAAKA-KVKRVVFTSIGFVMNPNRDTQAIVDENCWSDLDFCK TKNWYCY GKMI AEQSAWEIAKAKGVD OOnm -
270
280
290
300
310
320
330

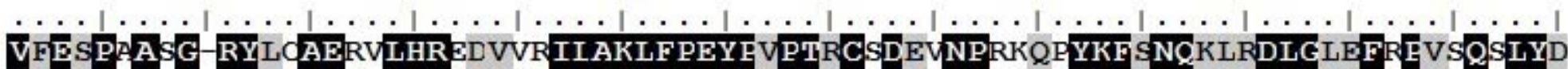
VYEAPYA G -RYIOAESTLHR GELCR IIAKLF PEY P ITKCKDDVNPPVTGYKFTNCRLKDLCMDFVEVIQGLYE VLEA PGA GGRYVOAERTLHR GELCR ILVGLF PEY I PTRCKDQVNPPKKGYKFTNQPLKDLG IKFIPVHEYLYE VFESPFASG-RHLOAERVLHR EDVVR II AKLF PEY FVPARCSDEVNPRKQPYKF SNOLLDLGLCFREVSQSLYD VYEA PSA SG-RYLI AE SARHR GEVVEIIAKLF PEY PI PTKCKDEKNPRAKPYKFTNQK IKDLGLEFTSTKQSLYD VYEAPSASG-RYIIAETALHRGEVVEIIAKFF PEY PIPTKCSDEKNPRAKPYKFTTQKIKDLGLEFKEIKQSLYE 


\section{Figure 3 (on next page)}

Figure 3 


\section{Figure 4 (on next page)}

Figure 4 

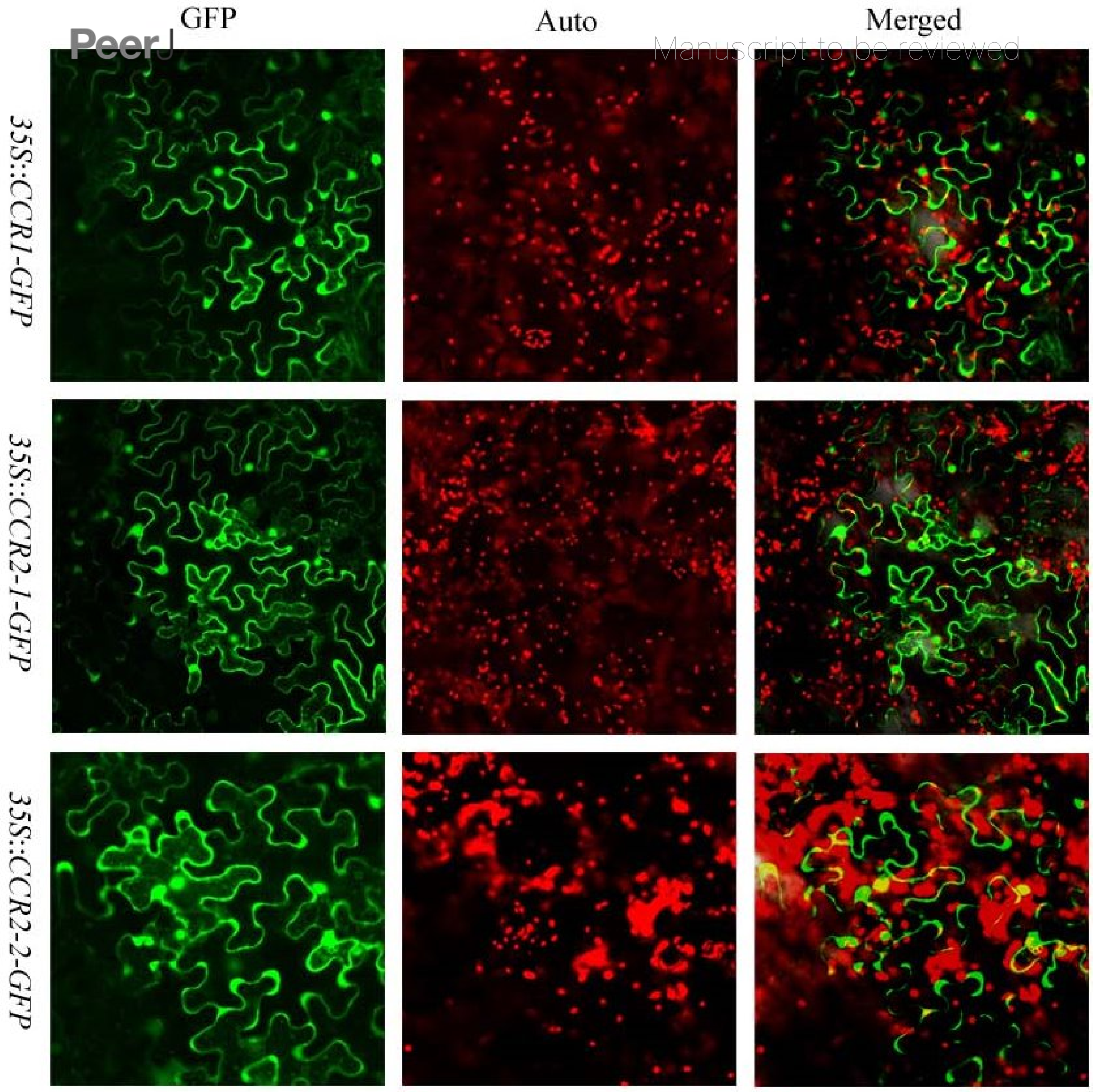

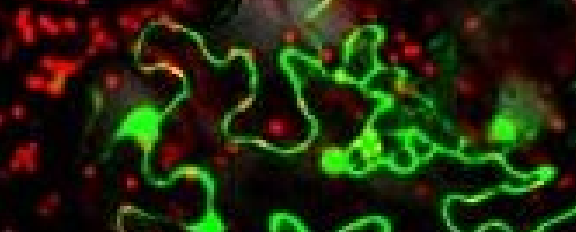

Manus cripteto be-reviewred
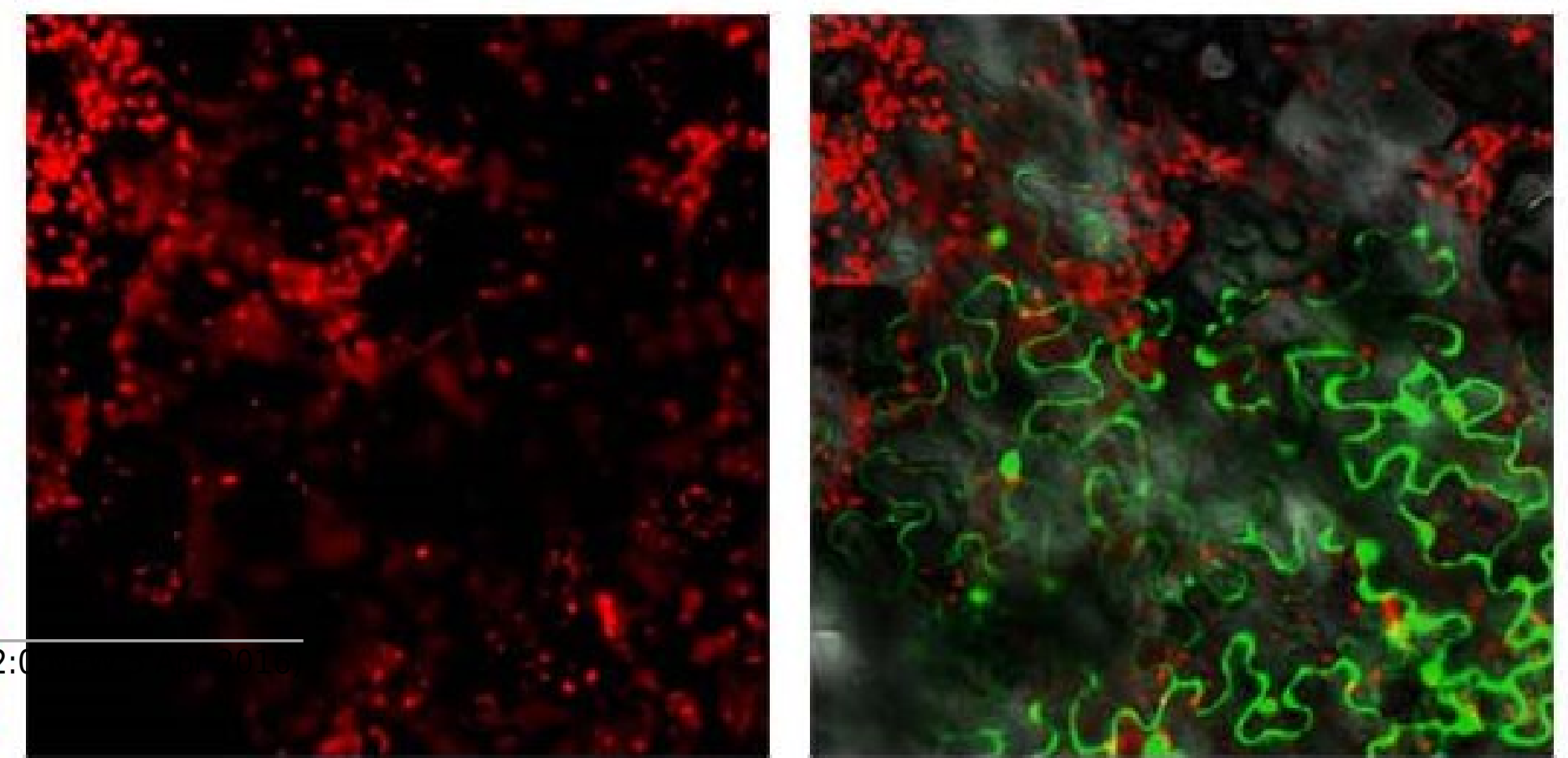


\section{Figure $\mathbf{5}$ (on next page)}

Figure 5 


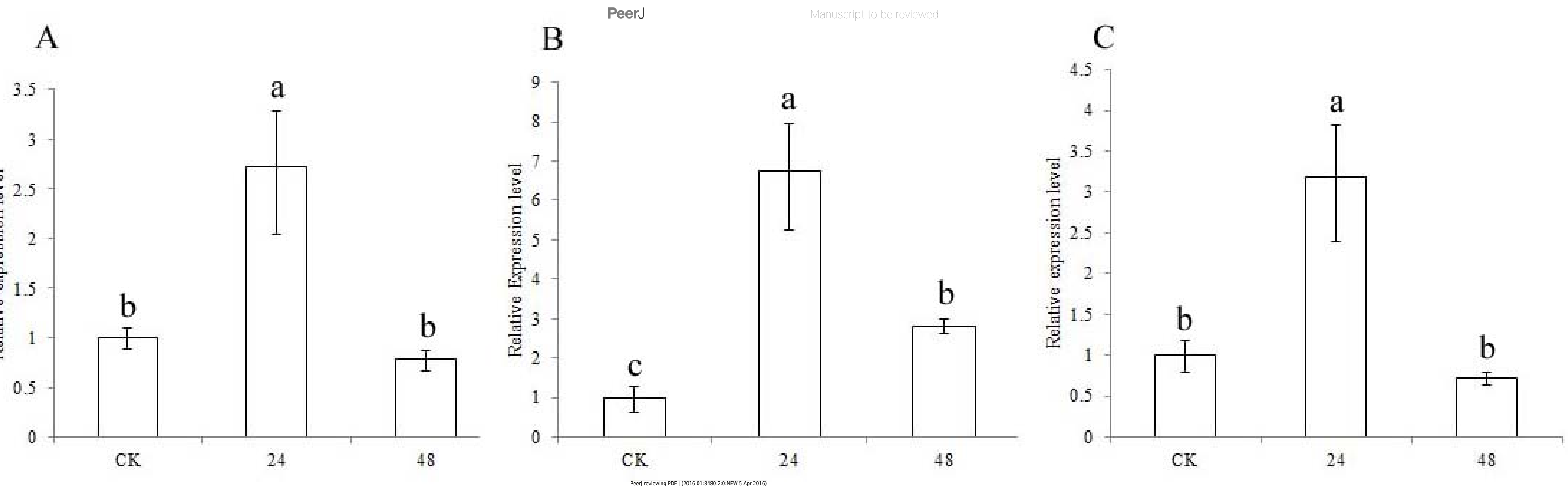




\section{Figure $\mathbf{6}$ (on next page)}

Figure 6 


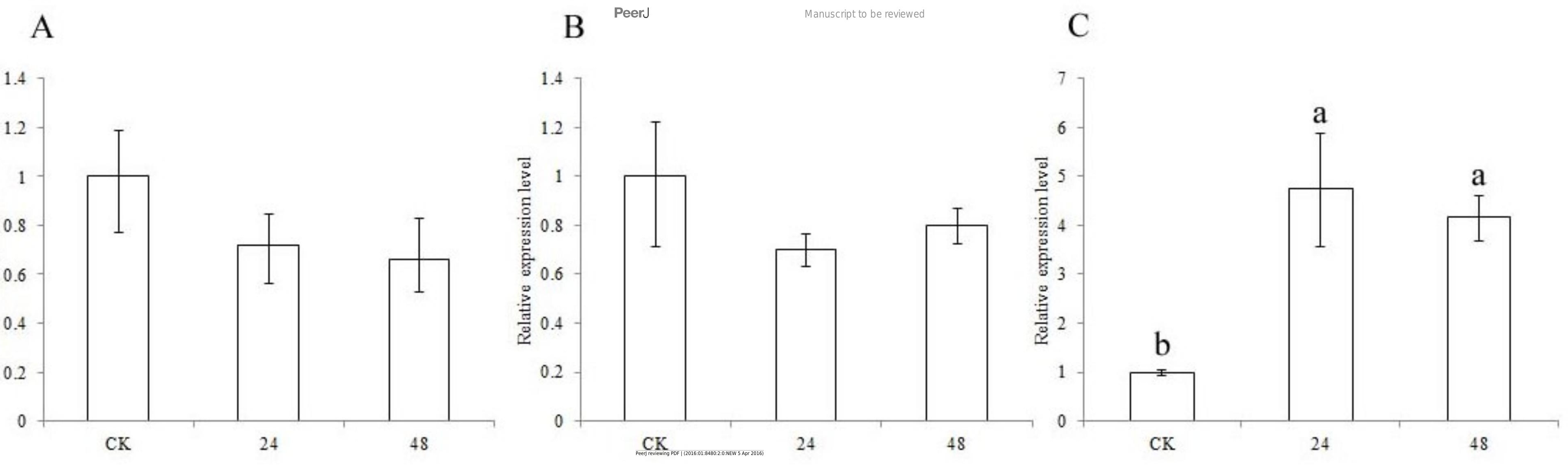




\section{Figure 7 (on next page)}

Figure 7 


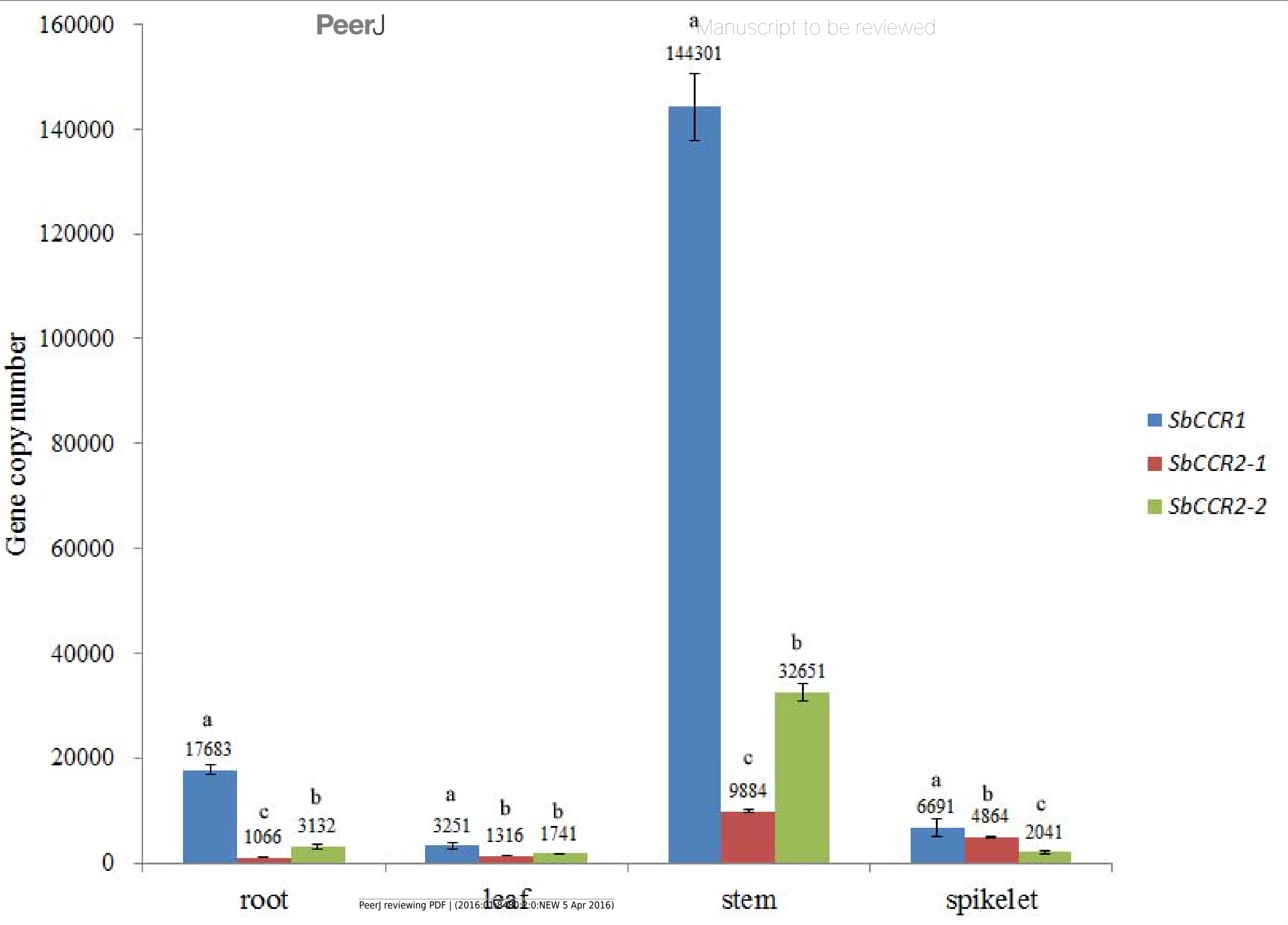




\section{Table $\mathbf{1}$ (on next page)}

The primers used in the research 
1 Table 1 The primers used in the research

\begin{tabular}{cccc}
\hline Primer name & Forward primer & Reverse primer & Usage \\
\hline GSbCCR1 & CATCACTCGACCGCACATAC & CAGCCAGCGAACAAACACTA & Gene \\
GSbCCR2-1 & TCAGACTAATAACCCGCCTAG & GCAGTATCAGCGTTGGAAA & cloning \\
GSbCCR2-2 & TCTTTCCGCTTCCACCGAT & GACTGCCAAAATTAATAACCAA & \\
SSbCCR1 & CGGACTAGTATGACCGTCGTCGAC & TCCCCCGGGCGCACGGATGGCGA & Subcellular \\
& & T & localization \\
SSbCCR2-1 & CGGACTAGTATGCCAACAGCAGAG & CGCGGATCCTGATTTGTGGAGTTG & \\
SSbCCR2-2 & CGGACTAGTATGGCCGTCGTCGTG & TCCCCCGGGAAGTTTTGAAATCA & \\
& & A & \\
YSbCCR1 & ATGCTGCTCGAGAAGGGATACAC & GTTCTTCGGGTCATCTGGGTTC & qRT-PCR \\
YSbCCR2-1 & ACCCACAAAGTGCAAGGACGAC & CCGCTGGTTCGTGAACTTGTATCC & \\
YSbCCR2-2 & GGAGTACCCTATTCCGACAAGGTG & GCACTGGCGTGAACTTGATTCC & \\
\hline & & &
\end{tabular}


Table 2 (on next page)

Molecular characteristics of CCR genes in Sorghum bicolor 
Table 2 Molecular characteristics of CCR genes in Sorghum bicolor

\begin{tabular}{cccccc}
\hline Name & Gene number & CDS length & Peptide residue & Theoretical Mw (kDa) & $\begin{array}{c}\text { Theoretical } \\
\text { PI }\end{array}$ \\
\hline SbCCR1 & Sb07g021680 & 1549 & 374 & 40.24 & 5.47 \\
SbCCR2-1 & Sb02g014910 & 1268 & 346 & 38.21 & 6.50 \\
SbCCR2-2 & Sb04g005510 & 1378 & 343 & 37.95 & 8.79 \\
\hline
\end{tabular}

2

3 